\title{
\begin{tabular}{l|l} 
pcori $).$ & PATIENT-CENTERED OUTCOMES RESEARCH INSTITUTE \\
RESEARCH SUMMARY
\end{tabular}
}

\section{Stroke and Blood Clot Risk in Transgender Women Taking Hormones}

Principal investigator

Michael Goodman, MD, MPH
Organization

Emory University

\section{What was the research about?}

People who are transgender have a gender identity that differs from the male or female sex assigned to them at birth. Some transgender people get therapies to change their bodies to match their gender identity. For transgender women, therapy may include taking hormones such as estrogen.

This research project earlier showed that transgender women who took estrogen had a higher risk of blood clots and strokes than men and women who weren't transgender. In this study, the research team looked further to learn how different types of hormone therapy affect those risks. The team looked at whether taking estrogen as a pill, a shot, or a patch affected the risk. They also looked at the risks of taking estrogen along with different medicines that decrease testosterone.

\section{What were the results?}

Overall, transgender women who took estrogen had a higher risk of blood clots and strokes than men and women who weren't transgender.

Compared with men and women who weren't transgender, the risk of blood clots was

- Higher in transgender women who took estrogen as pills

- About the same in transgender women who took estrogen as shots or patches
- Lower in transgender women who took spironolactone rather than other medicines used to lower testosterone

Also, the risk of strokes was

- Higher in transgender women who took estrogen as pills, shots, or patches

- About the same no matter which medicine transgender women took to lower testosterone

\section{Who was in the study?}

The research team looked at the health records of 3,325 transgender women and 62,033 men and women who weren't transgender. All were members of Kaiser Permanente health systems in California. Of these, 55 percent were White, 12 percent were Asian, and 6 percent were Black. Also, 20 percent were Hispanic, and 64 percent were ages 18-36.

\section{What did the research team do?}

Using health records, the research team compared the risk of blood clots and strokes in transgender women taking hormone therapy versus men and women who weren't transgender.

Transgender people, advocates, doctors, and health system staff worked with the research team during the earlier study.

\section{What were the limits of the study?}

This study looked at health records from patients in one health system in one state. Hormone therapy may 
vary across people and health providers. As a result, findings may differ for other patients.

Future research could continue to look at the risks of blood clots and strokes among transgender women. Studies could look at standardized hormone therapy.

\section{How can people use the results?}

Transgender women and their doctors can consider the results when starting hormone therapy. They can also look for signs of blood clots and strokes when taking hormone therapy.

To learn more about this project, visit www.pcori.org/Goodman356. 